\title{
Design and Numerical Study of Micropump Based on Induced Electroosmotic Flow
}

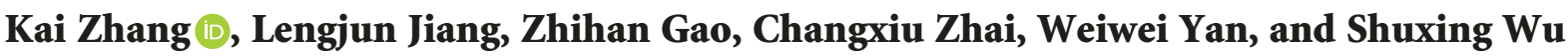

Institute of Fluid Engineering of China Jiliang University, Hangzhou 310018, China

Correspondence should be addressed to Kai Zhang; zkzb3026@yahoo.com

Received 1 February 2018; Accepted 5 April 2018; Published 9 May 2018

Academic Editor: Xiaoke Ku

Copyright (C) 2018 Kai Zhang et al. This is an open access article distributed under the Creative Commons Attribution License, which permits unrestricted use, distribution, and reproduction in any medium, provided the original work is properly cited.

Induced charge electroosmotic flow is a new electric driving mode. Based on the Navier-Stokes equations and the PoissonNernst-Planck (PNP) ion transport equations, the finite volume method is adopted to calculate the equations and boundary conditions of the induced charge electroosmotic flow. In this paper, the formula of the induced zeta potential of the polarized solid surface is proposed, and a UDF program suitable for the simulation of the induced charge electroosmotic is prepared according to this theory. At the same time, on the basis of this theory, a cross micropump driven by induced charge electroosmotic flow is designed, and the voltage, electric potential, charge density, and streamline of the induced electroosmotic micropump are obtained. Studies have shown that when the cross-shaped micropump is energized, in the center of the induction electrode near the formation of a dense electric double layer, there exist four symmetrical vortices at the four corners, and they push the solution towards both outlets; it can be found that the average velocity of the solution in the cross-flow microfluidic pump is nonlinear with the applied electric field, which maybe helpful for the practical application of induced electroosmotic flow in the field of micropump.

\section{Introduction}

The huge technological advances have made people's demand for technology products continue to move toward the direction of portability, miniaturization, and intelligence. With the improvement of living standards, more and more attention is being paid to health problems and the accuracy of the results, and the monitoring methods have also been set at higher standards. At this moment, the microfluidic chip is a good choice for further development and popularization of real-time diagnostic technology.

The so-called microfluidics chip refers to a chemical or biological lab built on a chip that is only a few square centimeters. It integrates the processes of biological and chemical reactions and separation and detection into microchannels, while using the design of microchannel networks for microfluidic control and transport and ultimately enables various functions in chemical or biological laboratories, that is, lab on a chip. Much research has been made under the leadership of a large number of scholars such as Lin Bingcheng, Qin Jianhua, and so on, all of which contributed to the development of microfluidic chip technology in China and even in the world. The internationally renowned magazine Lab on a Chip even published a special album titled "Focus on China" on the 10th anniversary of its founding, which is to affirm the important contribution made by Chinese scholars to the research of microfluidic technology.

The delivery mixing, reaction, separation, and control of microfluidics are key components of microfluidics system. Because of its small characteristic scale, most of the fluid flowing in the microchannels is laminar. Particles, droplets, or bubbles generally within the microchannels belong to the field of low Reynolds number flow theory [1-3]. Due to the sharp decrease of the volume to surface area ratio, the study found that the laws and phenomena of fluid movement at the microscale are different from the macro environment; the continuity equation in the three equations of hydrodynamics may no longer be suitable for 
use in microfluidics [4]. With the reduction of the characteristic scale of the study of fluid motion, a new flow effect was found. The coupling of the electric field force, the flow field, the temperature field and the ion motion within the microchannel, the electroosmotic flow, electrophoresis, induced electroosmotic flow, and other electrical phenomena can be used to achieve microfluidic (microparticle) transport and control $[5,6]$. Electrokinetic phenomena in microfluidics are caused by the interaction between the applied electric field and the diffusion layer in the electric double layer. More electrokinetic phenomena studied at present include electrophoresis, dielectric electrophoresis, electroosmotic flow, and induced electroosmotic flow. Electrokinetic phenomena can be divided into linear (electrophoresis and electroosmotic flow) and nonlinear (dielectric electrophoresis, electrophoresis, and induced electroosmotic flow) electrokinetic phenomena according to whether the zeta potential in the electrokinetic phenomenon changes with an applied electric field. In this paper, the phenomenon of induced electroosmotic flow is used.

Induced electroosmosis (ICEO) is a phenomenon driven by electrostatic forces under applied electric field and is a variant of the electroosmotic phenomenon [7]. The phenomenon of induced electroosmotic flow mainly depends on the interaction of polarizable solids with an applied electric field to generate an electromotive phenomenon. The induced potential on the polarizable surface is critical to the induced charge electroosmotic flow. The magnitude of its zeta potential is related to the applied electric field. The earliest induced electroosmotic flow was discovered by Romans et al. at the end of the 20th century. Subsequently, in 2004, Bazant and Squires perfected the relevant theory and formally proposed the concept of inducing electroosmotic flow. And the study of the mixing [8] and transporting of the fluid in the simple microchannel is accomplished by using this theory. By 2005, Levitan used experimental methods to confirm the correctness of the basic model of induced electroosmotic flow.

The induced charge electroosmotic flow (ICEOF) has been studied and applied to the microfluidic systems extensively in the last two decades. The phenomenon is used by $\mathrm{Wu}$ and $\mathrm{Li}$ to realize the function of fluid mixing and flow regulation in microfluidic chips; Zhao and Bau used induced electroosmotic flow to enhance chaotic flow to improve the mixing efficiency of microfluidics; Yariv, Bau, and $\mathrm{Li}$ et al. gave attention and conducted preliminary studies on inducing particle-wall effect in electroosmotic flow; Peng then experimentally found that the higher the zeta potential of the electrical double layer around the surface of the polarizable solid, the more particles agglomerated; demonstrating the feasibility of using micronanoparticle manipulation to induce electroosmosis. Harbin Institute of Technology, Peng and Jia innovated the use of ITO conductive glass as the electrode, based on the principle of induced electroosmotic flow and implementation of micronanoparticle manipulation.

Compared with the classical electroosmotic flow, the induced electroosmotic flow can obtain a higher driving speed under the same voltage, so we design a micropump [9-11] based on it, which can be applied to the driving of microfluidic chip.

\section{Theoretical Analysis}

2.1. Governing Equations and Boundary Conditions. In this study, the theoretical model is based on the NavierStokes equation [12] of viscous fluid flow, combined with the Poisson-Nernst-Planck (PNP) ion transport equation. The model can successfully predict new phenomena when the applied voltage is too small to disrupt the salt concentration.

As the flow is considered steady and incompressible, the governing equations are shown below:

$$
\begin{aligned}
\operatorname{Pe}^{-1} \nabla \cdot\left(\mp n_{+} \nabla \varphi-\nabla n_{ \pm}\right)+\vec{v} \cdot \nabla n_{ \pm} & =0 \\
\nabla \cdot(\operatorname{Pe} \vec{v} q) & =\nabla \cdot(c \nabla \varphi+\nabla q) \\
\nabla \cdot(\operatorname{Pe} \vec{v} c) & =\nabla \cdot(q \nabla \varphi+\nabla q) \\
2 \lambda_{0}^{2} \nabla^{2} \varphi & =-q \\
\nabla \cdot \vec{v} & =0 \\
\operatorname{Re}(\vec{v} \cdot \nabla \vec{v}) & =-\nabla p+\nabla^{2} \vec{v}-\nabla^{2} \varphi \nabla \varphi
\end{aligned}
$$

where $\lambda_{0}^{2}=\varepsilon_{f} k T /\left(2 z^{2} e^{2} a_{\mathrm{p}}^{2} n_{\infty}\right), \mathrm{Pe}=U a_{\mathrm{p}} / D, \mathrm{Re}=u a_{\mathrm{p}} / v=$ $\mathrm{PeSc}$. The other variables are the characteristic speed $u$, the characteristic length $a_{\mathrm{p}}$, the kinematic viscosity $v$, the dielectric constant $\mathcal{E}$, the valence of ions $z$, the absolute temperature $T$, the ion concentration $n_{\infty}$, the diffusion coefficient $D$, and Boltzmann's constant $k$.

Equations (2)-(4) are solved to obtain ion concentration and density distribution, and then (5) and (6) are solved to get the information of flow field. The zeta potential in the electroosmotic flow is induced by an applied electric field, and the magnitude of the potential depends on the applied electric field. According to the relevant theoretical study, it is found that the induced tangent slip velocity of the electric double layer on the polarizable solid surface in the electroosmotic flow is

$$
u_{\mathrm{ICEO}}=\frac{\varepsilon \varepsilon_{0} r}{\mu} E^{2}
$$

where $\varepsilon$ is the dielectric constant, $\varepsilon_{0}$ is the dielectric constant of vacuum, $r$ is the radius, $\mu$ is the dynamic viscosity, and $E$ is the applied electric field strength.

2.2. Zeta Potential Verification. Induced charge electroosmosis flow (ICEOF) phenomenon, which is caused by the interaction between the applied electric field and the electric double layer formed on the polarizable surface, and zeta potential changes on the polarizable solid are shown in Figure 1 . 


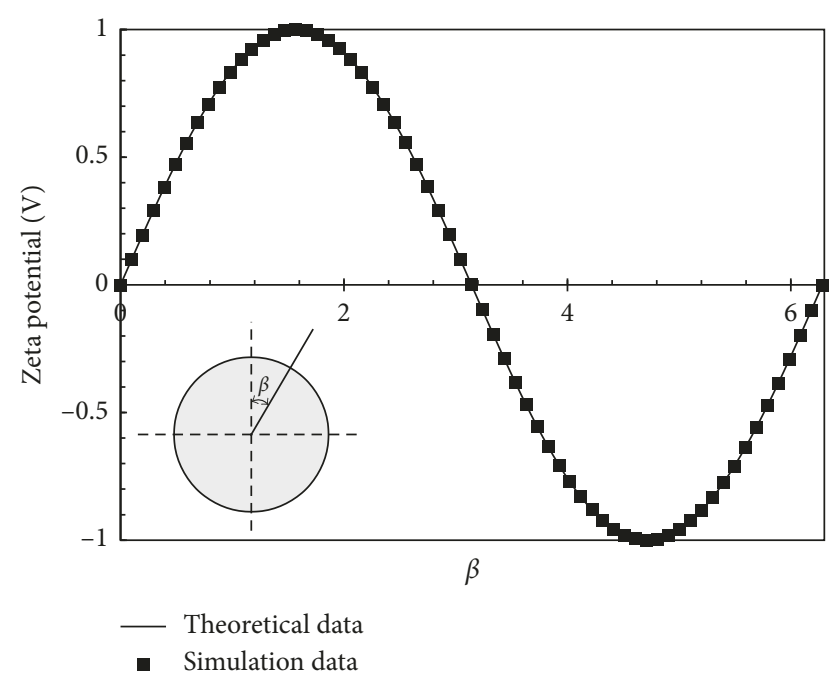

FIGURE 1: Zeta potential on the polarizable solid surface under the electric filed of $E_{0}$.

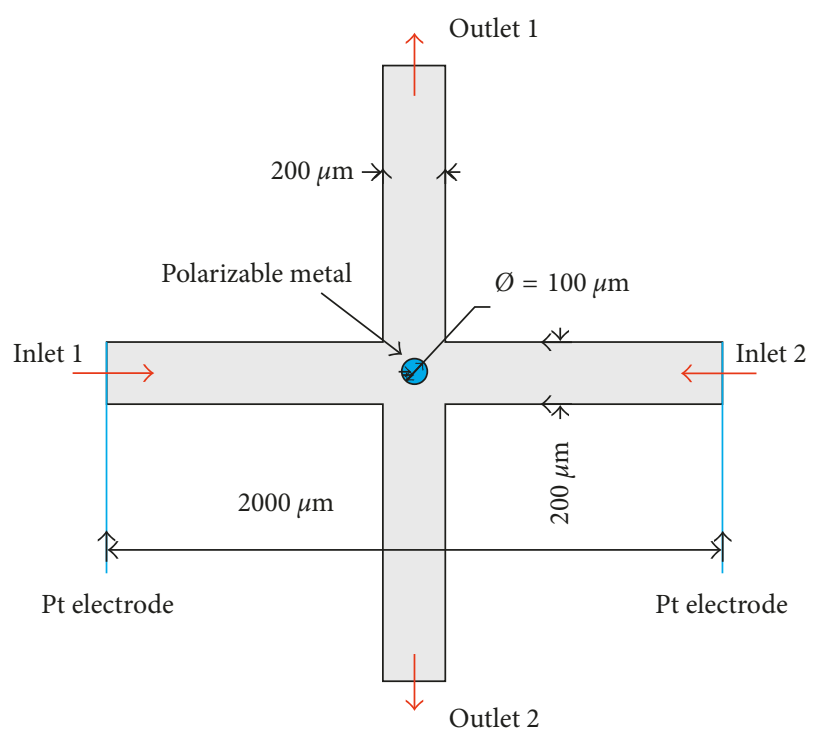

Figure 2: The geometry of micropump.

Under the two-dimensional uniform electric field, the analytical formula of zeta potential at ideal polarizable cylindrical surface is shown below:

$$
\zeta=2 E_{0} a \cos \beta .
$$

\section{Numerical Simulation}

3.1. Model and Boundary Conditions. As shown in Figure 2 of the cross-shaped induced electroosmotic micropump, a cylindrical polarizable solid is embedded in the middle of the cross-shaped channel. The distance between the energized electrodes is $L=2000 \mu \mathrm{m}$, the width of the microchannel is $W=200 \mu \mathrm{m}$, and the diameter of a circular polarizable solid is $\phi=100 \mu \mathrm{m}$. Using the Gambit software to mesh the $2 \mathrm{D}$ micropump model and pass the grid

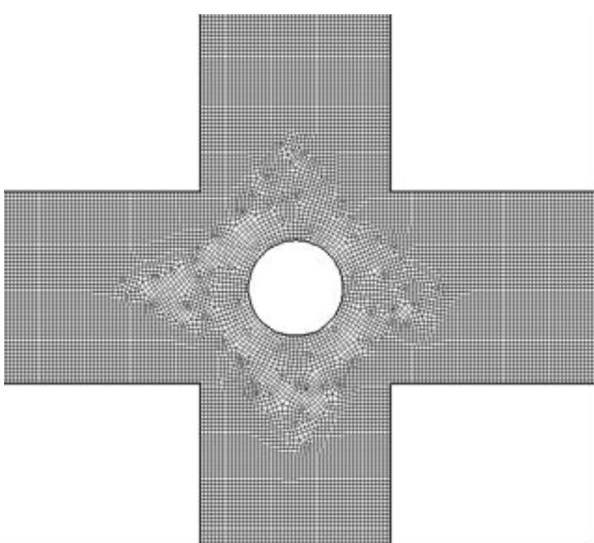

Figure 3: Mesh division of cross micropump.

independency verification, the cross geometry model of micropump is shown in Figure 3, and the total number of grids finally confirmed is 20,000 .

The model's boundary conditions are set as follows:

(1) Boundary conditions of the surface potential

Inlet and outlet: $\varphi_{\text {inlet-1 }}=\varphi_{a}, \varphi_{\text {inlet-2 }}=0 \mathrm{~V}$;

$\varphi_{\text {outlet-1 }}=\varphi_{\text {outlet }-2}=0 \mathrm{~V}$;

The surface of polarizes solid: $\partial \varphi / \partial \vec{n}=-(\partial c / \partial \vec{n}) / q$.

(2) Boundary conditions of ion concentration

Inlet and outlet: $c=2$;

Side wall: $c=2$;

The surface of polarized solid: $\partial c / \partial \vec{n}=-q(\partial \varphi / \partial \vec{n})$.

(3) Boundary conditions of ion density

Inlet and outlet: $q=0$;

Side wall: $q=0$;

The surface of polarized solid: $\partial q / \partial \vec{n}=-c(\partial \varphi / \partial \vec{n})$.

In this simulation, the flow field, the applied electric field, and the zeta potential control equation of the wall surface of the polarizable obstacle are shown in (4). The water used in the solution medium is related to the physical parameter: $\varepsilon_{r}=80, \varepsilon_{0}=8.85 e-12 \mathrm{C} \cdot \mathrm{V}^{-1} \mathrm{~m}^{-1}, \mu=1.003 e^{-3} \mathrm{~kg} \cdot \mathrm{m}^{-1} \mathrm{~s}^{-1}$, $\rho=998.2 \mathrm{~kg} / \mathrm{m}^{3}$.

3.2. Results and Discussions. First of all, the electric field of the cross channel is analyzed. In the simulation, an additional electric field is added to the two inlets to generate an electric field from the positive electrode to the negative electrode in the solution medium in the microchannel, as shown in Figure 4. At the same time, under the action of an applied electric field, the centrally located polarizable electrode is polarized, and the opposite ion in the adsorption solution forms a close-packed charge layer on the surface, eventually producing an electric double layer near the surface. The potential is the zeta potential, and the charge density around the polarizable solid is shown in Figure 5. In the program, the negative terminal defaults to zero, so the potential and charge in the positive direction will be more dense, but after the power is applied, an electric field will be generated between the positive and negative electrodes.

Therefore, when the center of the polarizable solid surface produces an electric double layer under the action 


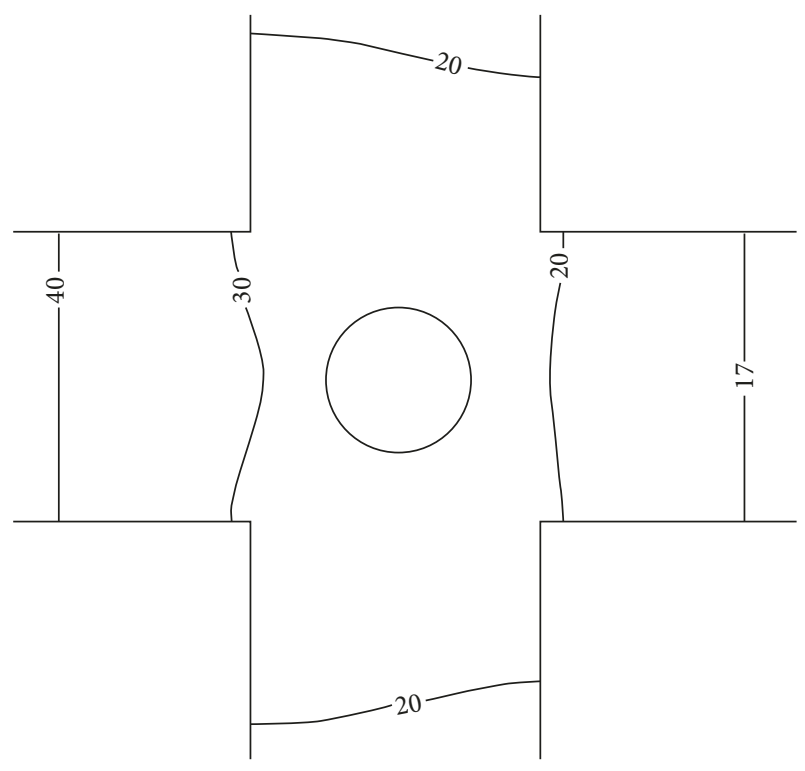

FIgURE 4: Voltage diagram in the cross channel.

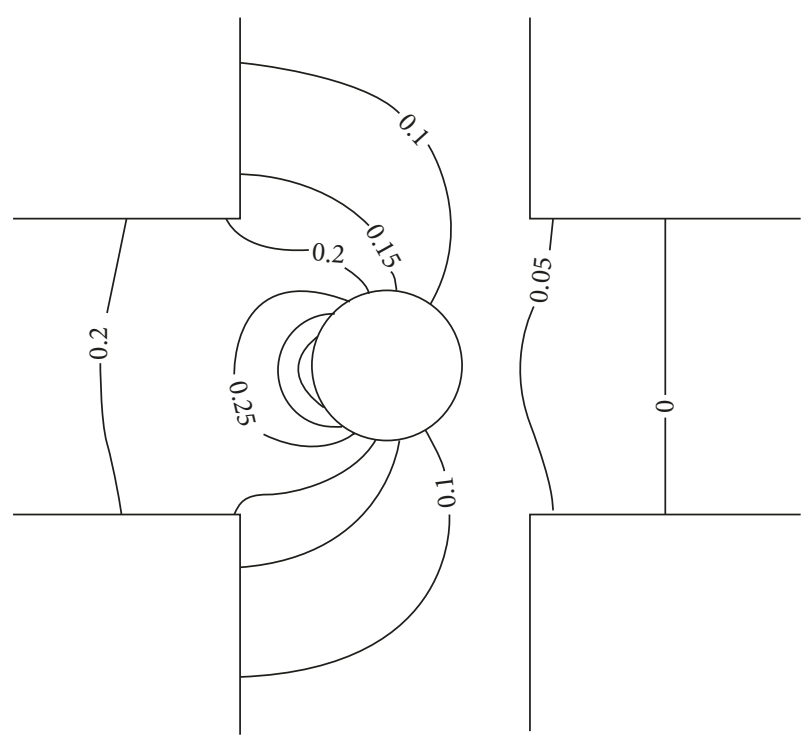

FIgURE 5: Charge density diagram the in cross channel.

of an applied electric field, the ions in the solution are attracted by the electric double layer, and finally the liquid is driven to form an induced electroosmotic flow. Figure 6 shows the micropump flow diagram of induced electroosmotic flow in the cross channel under different electric field intensities obtained from simulation. What can be seen from the diagram is that some of the fluids will flow along the polarizable solid surface from the left and right inlet to the outlet. Fluid at a distance farther away from the polarizable solids does not enter the exit channel but instead creates vortices around the polarizable solids. This is mainly due to the fact that the ion concentration in the diffusion layer in the electrical double layer is smaller in distance from the polarizable solid and less in drag force on the fluid driven by the external electric field, so that the fluid can not flow out from the outlet but do swirling movement in volatile solids around.

As the applied electric field increases, the shape of the vortex around the polarizable solid can also be found from Figure 6 above. When the voltage at the inlet is $\varphi_{a}=10 \mathrm{~V}$, the four vortices are basically at four corners and distributed evenly. With the increase of voltage, the four vortices around the polarizable solid gradually move toward the exit channel. When the voltage at the inlet is $\varphi_{a}=300 \mathrm{~V}$, it can be clearly seen that the four vortices basically entered the interior of the exit channel. At the same time, the distance between the two vortices of polarizable solids increases with increasing voltage. The above results show that the greater the voltage, the more easily the fluid flows into the outlet channel and also can result in a more efficient driving effect.

In general, the performance of a micropump is mainly measured by its microfluidic driving ability, which can be compared with the fluid velocity at the exit. This paper mainly simulates cross induced electroosmotic micropumps with a two-dimensional structure. Therefore, it is necessary to study the speed of its outlet. According to the simulation results, under the ideal conditions, micropump at the upper and lower exit has the same speed. Therefore, the speed of one of the outlets will be studied separately in this paper. Figure 7 shows the velocity profile at one outlet, where the vertical axis is the exit speed $v(\mathrm{~mm} / \mathrm{s})$ and the abscissa is the distance between the solution and the exit distance $l(\mathrm{~mm})$. As can be seen from the figure, the velocity at the outlet is parabolic, and the larger the voltage is, the greater the velocity is, and the driving effect of the micropump is better. When the voltage at the inlet is $100 \mathrm{~V}$, the maximum fluid velocity at the outlet of the electrical microchannel reaches $10 \mathrm{~mm} / \mathrm{s}$, and as the voltage increases, the drive speed increases faster and faster. This shows that the use of the micropump can produce a good driving effect.

Figure 8 shows the relation between the average velocity of single outlet and applied electric field strength, where the ordinate is the average speed at a single exit $v$ $(\mathrm{mm} / \mathrm{s})$ and the abscissa is the voltage at the power source $U(\mathrm{~V})$. It can be seen from the figure that the average speed at a single outlet is a quadratic nonlinear relationship with the power supply voltage, and when the power supply voltage is higher, the average speed of the micropump increases faster. When the voltage is greater than $100 \mathrm{~V}$, the average speed of the microchannel outlet at this time is already close to $10 \mathrm{~mm} / \mathrm{s}$. At this point, we linearly fit the numerical simulation results to get the cross structure of the micropump single-exit average velocity and applied electric field curve: $y=0.001 x^{2}-0.081 x+2.0618$.

\section{Conclusions}

In summary, the mechanism of induced electroosmotic flow is studied in depth. The analytical solution of induced zeta potential at polarizable solid surface is proposed by analyzing the governing equations of induced electroosmotic flow. Based on this theory, a UDF program suitable 


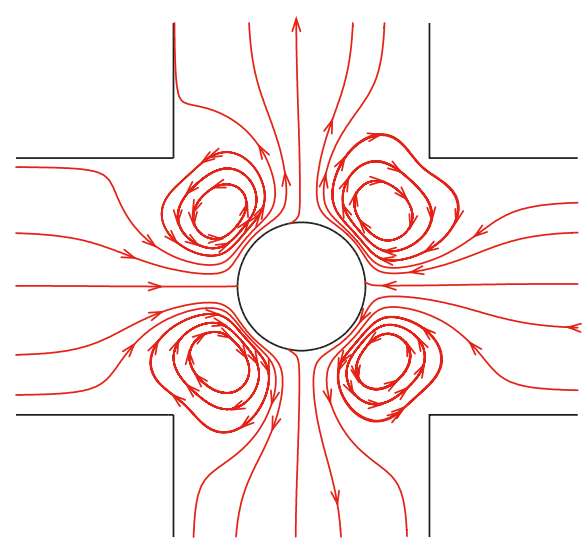

(a)

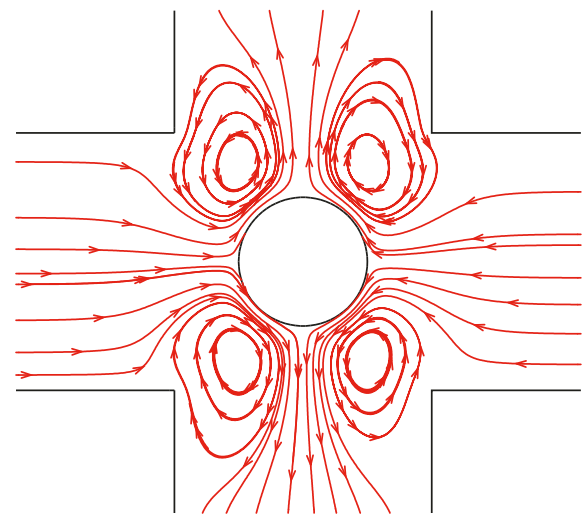

(c)

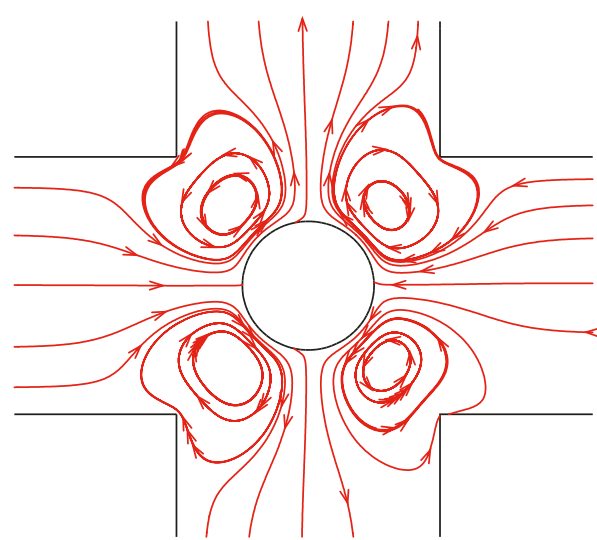

(b)

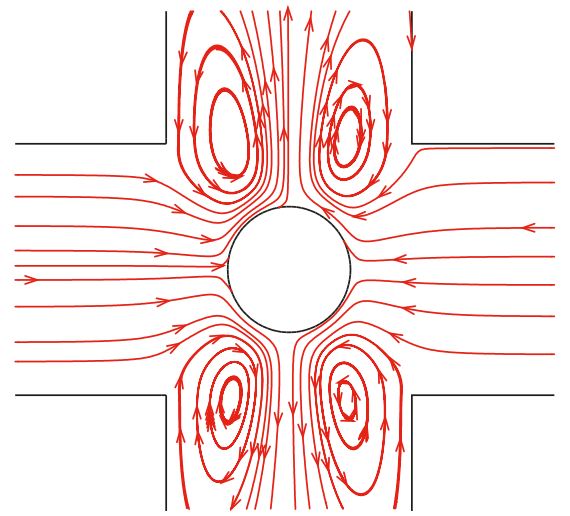

(d)

FIGURE 6: Streamline diagram of cross channel under different electric field intensities. (a) $\varphi_{a}=10 \mathrm{~V}$, (b) $\varphi_{a}=100 \mathrm{~V}$, (c) $\varphi_{a}=200 \mathrm{~V}$, and (d) $\varphi_{a}=300 \mathrm{~V}$.

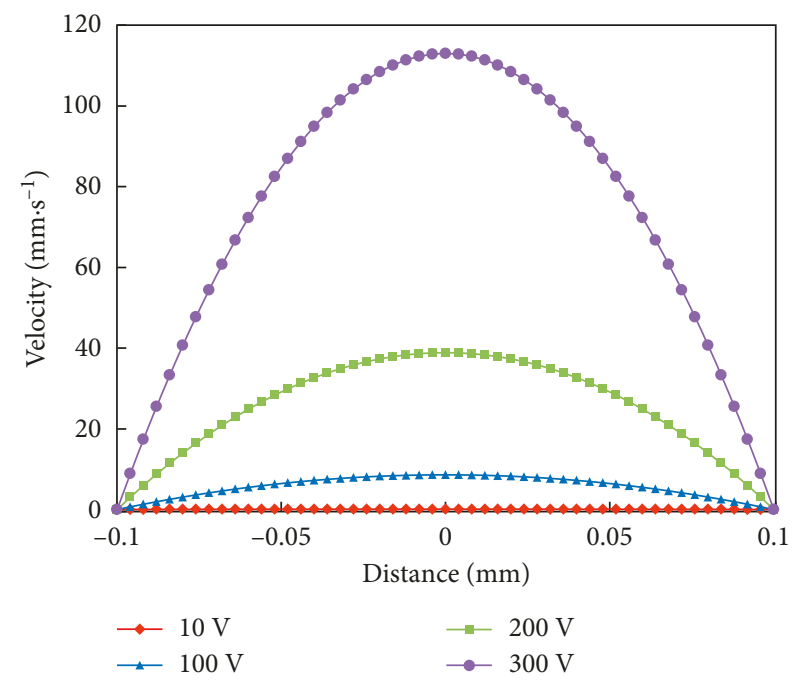

Figure 7: Velocity profiles at individual outlets at different voltages.

for induced charge electroosmotic flow simulation is developed. At the same time, the cross micropump driven by induced charge electroosmotic flow was designed, and the voltage, potential, charge density, and flow field of the induced micropump were obtained. The results show that

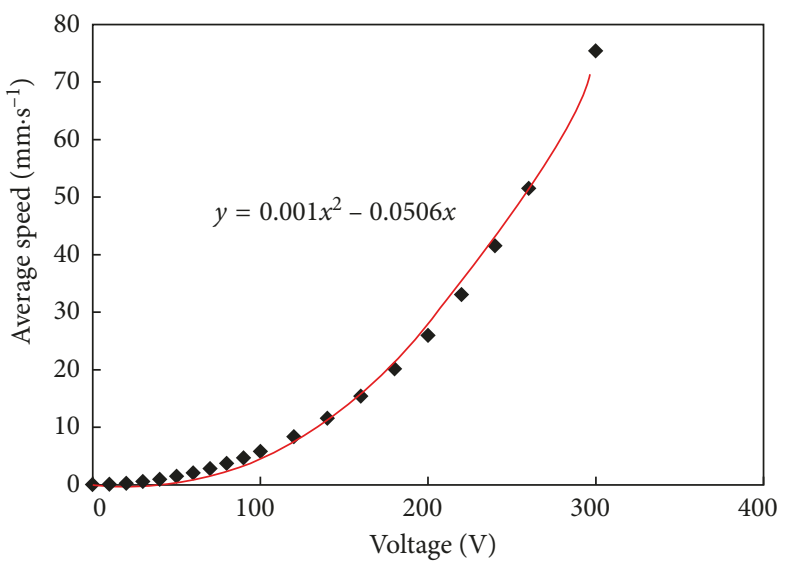

Figure 8: Relation between average velocity of single outlet and applied electric field strength.

the cross induced charge electroosmosis micropump has a nonlinear relationship with the applied electric field, which is more powerful than that of the traditional electroosmotic pump.

\section{Conflicts of Interest}

The authors declare that they have no conflicts of interest. 


\section{Acknowledgments}

This study was supported by the National Natural Science Foundation of China (11472260).

\section{References}

[1] J. Z. Lin, Y. L. Wang, P. J. Zhang, and X. K. Ku, "Mixing and orientation behaviors of cylindrical particles in a mixing layer of an Oldroyd-B fluid," Chemical Engineering Science, vol. 176, pp. 270-284, 2018.

[2] J. Lin, X. Pan, Z. Yin, and X. Ku, "Solution of general dynamic equation for nanoparticles in turbulent flow considering fluctuating coagulation," Applied Mathematics and Mechanics, vol. 37, no. 10, pp. 1275-1288, 2016.

[3] M. Z. Yu, J. Z. Lin, and T. Chan, "Effect of precursor loading on non-spherical $\mathrm{TiO}_{2}$ nanoparticle synthesis in a diffusion flame reactor," Chemical Engineering Science, vol. 63, no. 9, pp. 2317-2329, 2008.

[4] M. Z. Yu, Y. Y. Liu, J. Z. Lin, and M. Seipenbusch, "Generalized TEMOM scheme for solving the population balance equation," Aerosol Science and Technology, vol. 49, no. 11, pp. 1021-1036, 2015.

[5] K. Ward and Z. Hugh Fan, "Mixing in microfluidic devices and enhancement methods," Journal of Micromechanics and Microengineering, vol. 25, no. 9, p. 094001, 2015.

[6] K. Masilamani, S. Ganguly, C. Feichtinger, D. Bartuschat, and U. Rüde, "Effects of surface roughness and electrokinetic heterogeneity on electroosmotic flow in microchannel," Fluid Dynamics Research, vol. 47, no. 3, p. 035505, 2015.

[7] C. Canpolat, "Induced-charge electro-osmotic flow around cylinders with various orientations," Proceedings of the Institution of Mechanical Engineers, Part C: Journal of Mechanical Engineering Science, vol. 231, no. 21, pp. 4507-4066, 2017.

[8] C. Wang, Y. Song, X. Pan, and R. Dongqing Li, "A novel microfluidic valve controlled by induced charge electroosmotic flow," Journal of Micromechanics and Microengineering, vol. 26, no. 7, p. 075002, 2016.

[9] K. Bengtsson and N. D. Robinson, "A large-area, all-plastic, flexible electroosmotic pump," Microfluidics and Nanofluidics, vol. 21, p. 178, 2017.

[10] A. K. R. Lai, C. C. Chang, and C. Y. Wang, "Optimizing electroosmotic pumping rates in a rectangular channel with vertical gratings," Physics of Fluids, vol. 29, no. 8, p. 082002 , 2017.

[11] M. Gao and L. Gui, "A handy liquid metal based electroosmotic flow pump," Lab on a Chip, vol. 14, no. 11, pp. 18661872, 2014.

[12] M. Z. Yu, J. Z. Lin, and T. Chan, "Numerical simulation of nanoparticle synthesis in diffusion flame reactor," Powder Technology, vol. 181, no. 1, pp. 9-20, 2008. 


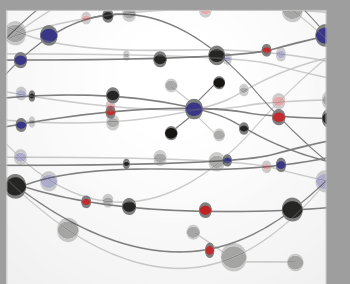

The Scientific World Journal
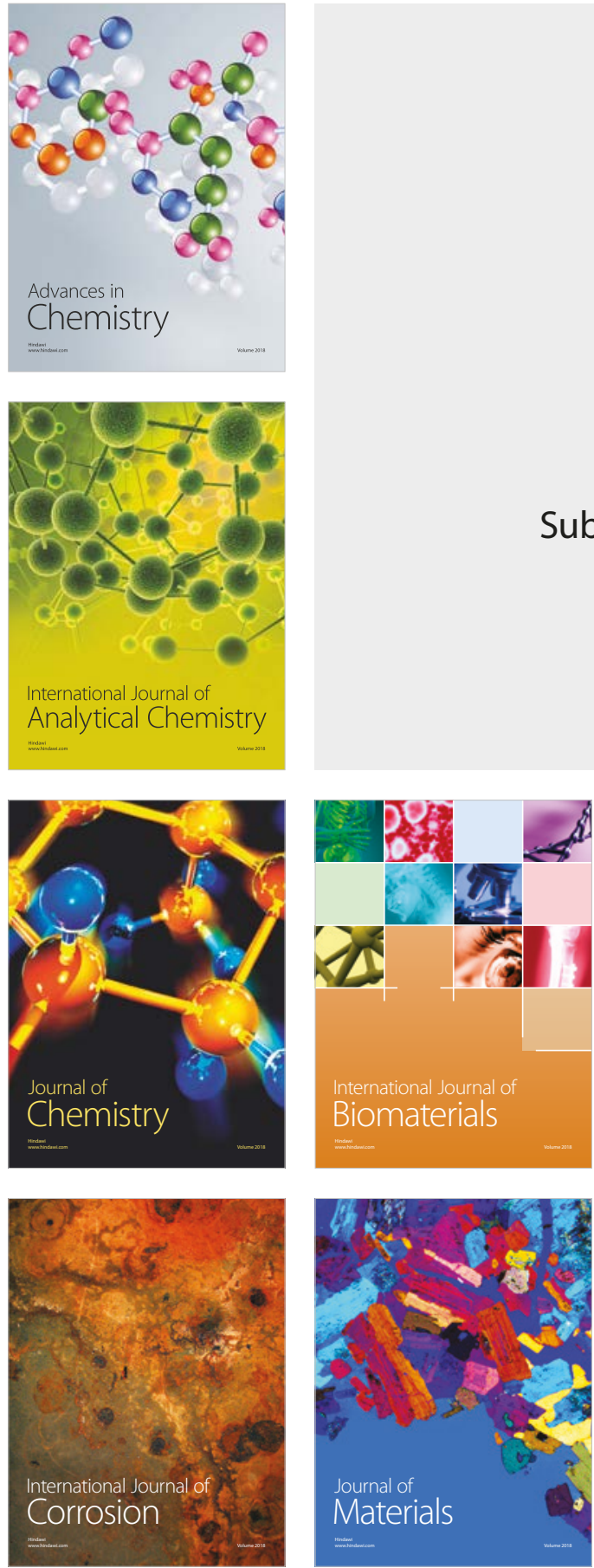

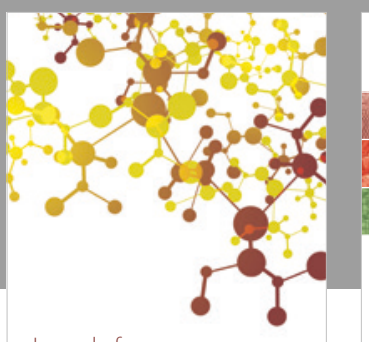

Journal of

Applied Chemistry
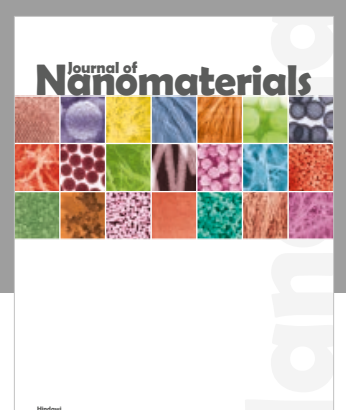

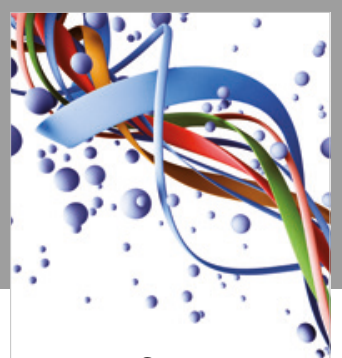

Scientifica

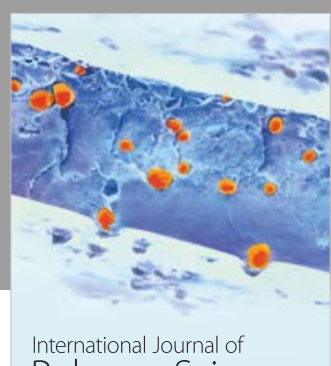

Polymer Science

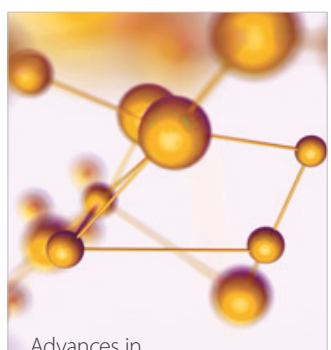

Physical Chemistry
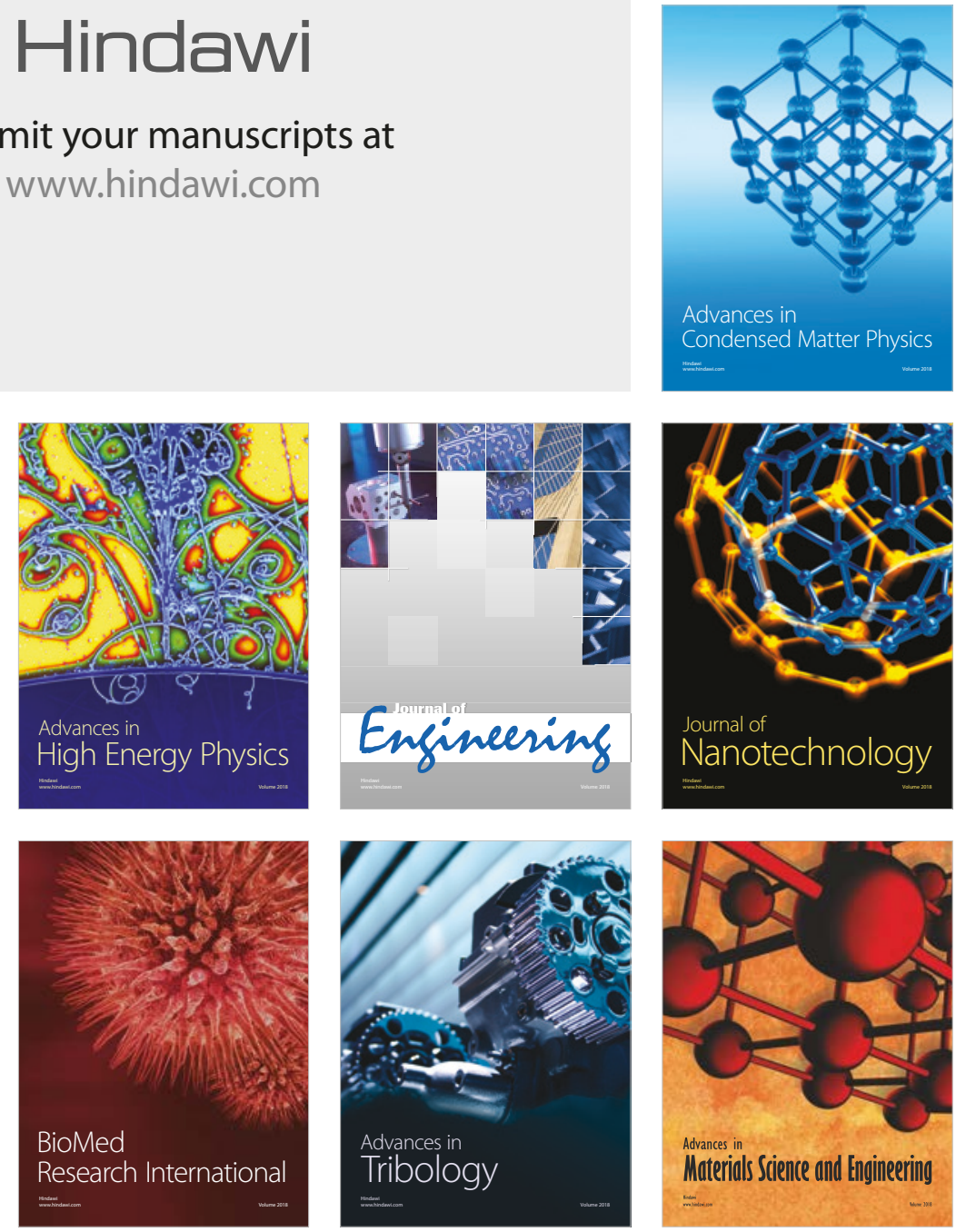